\title{
Predictions of Two Photon Absorption Profiles Using Time-Dependent Density Functional Theory Combined with SOS and CEO Formalisms
}

\author{
Sergio Tafur ${ }^{1,2}$, Ivan A. Mikhailov ${ }^{1}$, Kevin D. Belfield ${ }^{3,4}$, and Artëm E. Masunov ${ }^{1,2,3}$ \\ ${ }^{1}$ Nanoscience Technology Center \\ ${ }^{2}$ Department of Physics \\ ${ }^{3}$ Department of Chemistry \\ ${ }^{4}$ CREOL, College of Optics and Photonics, University of Central Florida, \\ 12424 Research Parkway, Suite 400, Orlando 32816, USA \\ amasunov@mail.ucf.edu
}

\begin{abstract}
Two-photon absorption (2PA) and subsequent processes may be localized in space with a tightly focused laser beam. This property is used in a wide range of applications, including three dimensional data storage. We report theoretical studies of 5 conjugated chromophores experimentally shown to have large 2PA cross-sections. We use the Time Dependent Density Functional Theory (TD-DFT) to describe the electronic structure. The third order coupled electronic oscillator formalism is applied to calculate frequency-dependent second order hyperpolarizability. Alternatively, the sum over states formalism using state-to-state transition dipoles provided by the a posteriori Tamm-Dancoff approximation is employed. It provides new venues for qualitative interpretation and rational design of $2 \mathrm{PA}$ chromophores.
\end{abstract}

Keywords: conjugated chromophores, two-photon absorption, time-dependent density functional theory, coupled electronic oscillators, sum over states, Tamm-Dancoff approximation, structure-activity relationship.

\section{Introduction}

Two-photon absorption (2PA) is an electronic excitation process involving simultaneous absorption of two photons. There are a wide range of 2PA applications, such as three dimensional data storage, photonic devices, lithographic micro-fabrication [1], quantum information technology [2], optical limiting, two-photon pumped lasing in organic chromophores and quantum dots [2, 3], in-vivo bioimaging, and cell-selective photo-dynamic therapy [3]. Most applications require chromophores with large 2PA cross-sections to minimize laser intensity requirements and prevent overheating of targets [1]. To design more efficient 2PA chromophores, it is important to understand their structure/activity relationships (SARs). Computer modeling of 2PA spectra facilitates understanding of these relationships and is becoming an important part rational approach that may accelerate progress in chromophore design [4]. Accurate predictions of 2PA spectral profiles would greatly assist in the design of more effective 2PA chromophores while eliminating poor candidates form the synthetic pipeline. The goal 
of this study is improvement in quantitative predictions of 2PA, as well as development of qualitative tools to understand the relations between the electronic structure of the chromophores and 2PA profiles.

In the past decades several research groups had made a strong effort aimed at the development of new compounds with large 2PA cross sections. The main guiding principle used in those studies involved electron transfer between electron-donor (D) and electron-acceptor (A) moieties attached symmetrically or asymmetrically to the $\pi$-conjugated bridge. Fluorene fragment in particular was found to be a good example of $\pi$-conjugated bridge due to highly delocalized $\pi$-system delocalized over the two benzene rings held together at nearly coplanar orientation by methylene bridge [5]. $D-\pi-A, D-\pi-D$, or $A-\pi-A$ molecular structures have been proposed and studied both theoretically and experimentally. In recent studies fluorene derivatives have been extended to $D-\pi-\pi-A$ and $A-\pi-\pi-A$ types with the aim of increasing 2PA absorption cross-sections [6-10]. However, the choice of functional groups and linkages the most appropriate for developing chromophores with the largest 2PA characteristics it is still under active investigation.

In order to accelerate the experimental efforts based on traditional trial and error approach, a quantitative understanding of the trends in dependence of 2PA crosssection on molecular structure would be clearly beneficial. Two major approaches had been used applied to accomplish that goal. First is based on essential state models (three-state, four-state, etc.). Parameters of these models (such as excitation energies and transition dipoles) are adjusted to fit experimental data. These parameters are then correlated with details of molecular structure ( $\pi$-conjugated chain lengths, donor/acceptor strengths, etc.). Another approach consists of quantitative prediction of 2PA cross-sections at chosen level of theory, followed by analysis of the physical principles of the major contributions into this property. The levels of theory, used for 2PA predictions cover the wide range.

In recent quantum chemical calculations performed on conjugated chromophores have shown that a substantial symmetric charge redistribution that occurs upon excitation may account for heightened sensitivity to 2PA events [6]. In their work Bredas et al. established a good agreement between the peak values of 2PA crossections measured with femto-second pulses and those calculated with semi-empirical intermediate neglect of differential overlap Hamiltonian with multi-reference double-configuration interaction (INDO-MRD-CI) scheme based methods. Aside from the donor-acceptor configuration of 2PA active chromophores, it was also established by Bredas et al. that increasing the length and charge transfer of the molecules results in an increase in 2PA crossections and may also result in a significant shift of 2PA to longer wavelengths [6]. Complementarily, Agren et al. theoretically studied four lowest excited states of $\pi$ conjugated systems experimentally produced and characterized by Kim et al. [10] and Ventelon et al. [9] using ab initio response theory. They showed that their theoretical findings were consistent with the correlation between large 2PA crossections and a $\pi$ center, but that though the one photon absorption (1PA) spectra was strongly correlated to the molecular length this was not always the case for 2PA in the visible domain [5]. At around this time it was also established by Fabian et al. that spectral absorption features are reasonably well reproduced by the approximate semi-empirical MO-CI methods based on the NDO (ZINDO/S), however time-dependent density functional response theory (TD-DFT) proved to be superior over semi-empirical methods [11]. 
Since then Hales et al. showed that 2PA spectra for symmetric and asymmetric fluorene derivative compounds exhibit intermediate resonant enhancement of nonlinearities, with an order of magnitude enhancement for asymmetric cases, when compared to degenerate 2PA. INDO-MRD-CI semi-empirical methods that implemented a simplified three level model were also shown to provide additional insight into the mechanisms governing 2PA events [12]. Several groups published works investigating the structure-activity relationships (SARs) responsible for the 2PA characteristics.

The conjugated chromophores selected as the subjects of this study are presented in Scheme 1. Theoretical models of these were derived by truncation of the aliphatic chains and replacing them with to methyl groups in the original experimental structures. The abbreviations of the model molecules and the systematic names of the corresponding experimentally studied ones are: BzFBz: 2,7-Bisbenzothiazolyl-9,9didecylfluorene; BzFDp: (7-benzothiazol-2-yl-9,9-didecylfluoren-2-yl)diphenylamine; DpFDp: 9,9-didecyl-2,7-bis(N,N-phenylamino)-fluorene; BzPFPBz: 2,7-Bis[4-(9,9didecylfluoren-2-yl)vinyl]-phenylbenzothiazole; DpPFPBz: \{7-[2-(4-Benzothiazol2ylphenyl)vinyl]-9,9 didecylfluorene-2yl $\}$ diphenylamine. These compounds were experimentally synthesized and characterized by Belfield et $\mathrm{al}$. as a model compounds for possible applications in two photon microfabrication, two photon photochemical transformations, non-destructive 3-D multiphoton fluorescence imaging, and photodynamic therapy [7, 12, 13]. They found large (600GM) cross-sections for BzFBz while studying the design of rigid-rod polymers while $2 \mathrm{PA}$ cross-sections of the polymers were reduced by aggregation [14]. Compound $\mathbf{B z F B z}$ additionally exhibited a large fluorescence quantum yield. The good chemical, thermal, and photochemical stability, combined with desirable one- and two-photon absorption and luminescence properties, stand out as characteristics of this chromophore as a promising material for two-photon based technologies [15]. Compound BzFDp has been previously implemented for in vivo 2PA biomedical imaging applications, as a fluorophore dye used for staining rat cardiomyoblast cells (H9c2), by Belfield et al. due to its high photostability, fluorescence quantum yield, and two-photon absorption cross-section over the tunable range of commercially available Ti:sapphire lasers [16]. Additionally, BzFDp has been investigated as a potential 2PA free-radical photo-initiator for three-dimensionally resolved polymerization, resulting in intricate micro-fabrication and imagining [17].

In semi-empirical wave function theory studies of $2 \mathrm{PA}$ active organics have been carried to a varying degree of success. The efforts put forward in these studies have circled around INDO (intermediate neglect of differential overlap) semi-empirical Hamiltonian models for molecular geometry optimizations and the implementation of the INDO Hamiltonian coupled to a MRD-CI (multi-reference double configuration interaction) formalism in the description of ground and excited states. The description of these states were then used to calculate ground and excited state energies, dipoles and transition dipoles [18] which in turn were implemented in the sum over states formalism (SOS) to calculate linear or nonlinear material response.

Recently, Time Dependent Density Functional Theory (TD-DFT) was successfully used to simulate 2PA electronic spectra in large conjugated molecules [19-21]. The coupled electronic oscillator (CEO) formalism performed well, after it was shown that the density matrix formulation of the time-dependent Kohn-Sham equations allows treatment of adiabatic TD-DFT on the same footing as the TDHF theory to an arbitrary order in the external perturbation [22]. This approach was shown to achieve superior 
accuracy for 2PA excitation energies, when compared to semi-empirical wave function theory methods. The tools used for implementation of the third order CEO at TD-DFT level are detailed below.

An alternative approach to using CEO in the framework of TD-DFT is to use state to state transition dipole moments calculated within TD-DFT, using second and third order response functions, and implement them in the SOS formalism [23, 24]. Difficulties that arise in the implementation of SOS are governed by the description/accuracy of the state to state transition dipole moments and excited state energies predicted by DFT. Cronstrand et al. and Kamada et al. present the possibility of using few states models derived from SOS to calculate the 2PA cross-section and arrive at a mechanism for 2PA $[25,26]$.

Of the compounds in Table 1, BzFDp and DpFDp have been previously studied theoretically by Hales et al. using INDO-MRD-CI with geometries optimized with an AM1 semi-empirical Hamiltonian. All their calculations were carried out on isolated molecules and showed a strong qualitative and quantitative agreement with experimentally generated spectra [12]. The three-level model developed in their study provided further insight into the mechanisms governing the nonlinear activity of 2PA active chromophores in relation to the description of the molecular states. A second study carried out by Day et al. implemented linear and quadratic response density functional theories to calculate the photo-physical properties of D- $\pi$-A molecules including BzFDp. Their comparison of a two-state approximation and with calculation of 2PA via the SOS method with the inclusion of higher energy states drew a conclusion that the inclusion of higher energy states was necessary in the description of 2PA [27, 28].

In this contribution we obtain approximate state-to-state transition dipole moments $\mu_{n m}^{i}$ within a TD-DFT formalism by implementing the a posteriori Tamm-Dancoff approximation (ATDA, introduced in Ref. [29]), as an approximation to second order DFT, and employ them to identify the essential states governing the 2PA process. We also validate ATDA results by using these $\mu_{n m}^{i}$ to evaluate the resonant 2PA crosssections with sum over state models (SOS) and compare them to CEO results as well as experimental values.

\section{Theory}

Time-Dependent Density Functional Theory (TD-DFT) was recently combined with the Coupled Electronic Oscillator (CEO) formalism to simulate 2PA electronic spectra in large conjugated molecules $[19,30]$. These and other 2PA predictions using TD-DFT [31] were shown to achieve quantitative agreement with experiment and higher-level $a b$ initio predictions. In this contribution we also use an alternative Sum Over States (SOS) approach, calculate state-to-state transition dipole moments $\mu_{n m}^{i}$ using the a posteriori Tamm-Dancoff approximation and employ them to identify essential states governing the 2PA process. We also validate ATDA by using these approximate $\mu_{n m}^{i}$ to predict resonant $2 \mathrm{PA}$ cross-sections with the SOS model and compare them to CEO results as well as experimental values.

In the 2PA-transition matrix approximation the 2PA cross-section is given by [25]: 


$$
\begin{gathered}
\sigma_{2 P A}=\frac{8 \pi^{3} \omega^{2}}{15 c^{2}} g(2 \omega) \sum_{a, b}^{3}\left(M_{a a} M_{b b}^{*}+2 M_{a b} M_{a b}^{*}\right), \\
M_{i j}=\frac{1}{2 \hbar} \sum_{k}^{N}\left(\frac{\mu_{f k}^{i} \mu_{k g}^{j}+\mu_{f k}^{j} \mu_{k g}^{i}}{\omega_{k g}-\omega-i \Gamma / \hbar}\right)
\end{gathered}
$$

here $\mathrm{g}(2 \omega)$ is the Lorentzian lineshape, and $\mu_{n m}^{i}$ are state-to-state transition dipole moments. This ATDA/SOS approach opens new venues for interpretation of 2PA properties in terms of molecular electronic structure and can be used for rational design of 2PA chromophores.

\section{Computational Details}

The chromophore molecules selected for this study are presented in Scheme 1. They were derived from experimentally studied ones by truncation of the aliphatic chains to methyl group. All molecular structures were optimized at HF/STO-3G theory level, which favors planar geometry of conjugated molecules and was shown [31] to give the best agreement for the bond lengths as compared to the results of X-Ray diffraction experiments for stylbene and its three derivatives. The optimized geometries were confirmed by the absence of imaginary frequencies in the following normal mode calculations. The single point energy and transition dipole calculations were performed at the TD-B3LYP/MIDIx level.

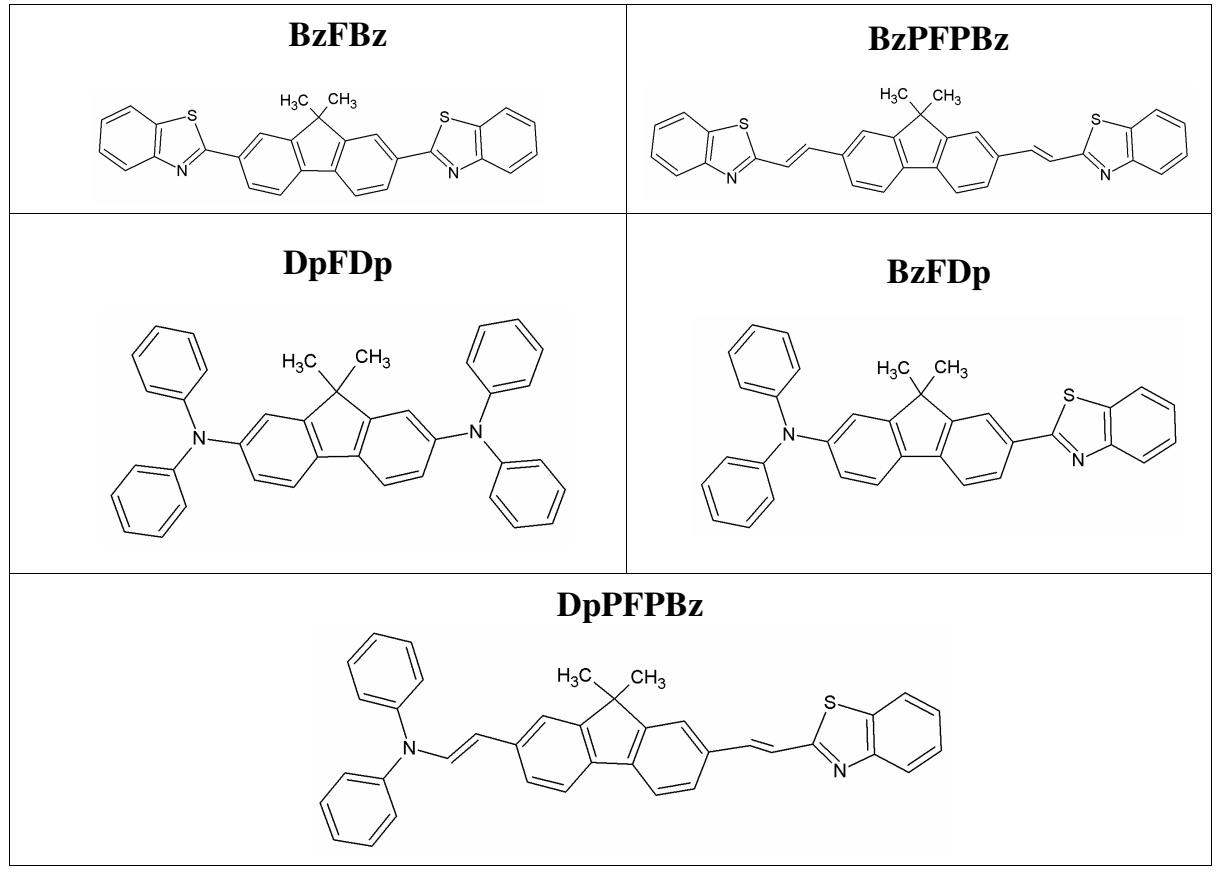

Scheme 1. Structural formulas of the molecules studied 
Transition density matrices for the lowest 24 excited states, as well as Kohn-Sham operators on these transition densities were printed out. Contributions of the second and third derivatives of the exchange-correlation potentials into Kohn-Sham operators, and operators on the pair combinations of transition densities were neglected. Commercially available computational program Gaussian98 [32] was modified as described in previous studies [19] in order to enable this printout. The frequencydependent orientationally averaged first- and third-order polarizability tensors were generated from the generated matrices using (1) and expressions implemented in CEO program [33]. The habitual empirical linewidth of $0.1 \mathrm{eV}$ was used for both 1PA and 2PA. To analyze the electronic structure of the excited states we used natural transition orbitals (NTO), which diagonalize the transition density matrix, and give the best representation of the electronic excitation in single-particle terms [34]. Graphical software XCrysDen [35] was used to plot NTOs.

\section{Results and Discussion}

We present 2PA resonant energies and cross-sections in Table 1. For two of the molecules, the profiles obtained with both SOS and CEO formalisms are presented in Fig. 1, along with linear spectra, and natural transition orbitals.

Table 1. Energies and cross-sections for the linear and 2PA absorbing states in the molecules studied

\begin{tabular}{|c|c|c|c|c|c|}
\hline State & $2 \mathrm{PA}_{\text {calc }}, \mathrm{GM}$ & $\begin{array}{c}\Delta \mathrm{E}_{\text {vertical }}, \\
\mathrm{eV}\end{array}$ & $2 \mathrm{PA}_{\text {exp }}, \mathrm{GM}$ & $\begin{array}{c}\Delta \mathrm{E}_{\text {exp }}, \\
\mathrm{eV}\end{array}$ & $\begin{array}{c}\lambda_{\text {exp }} \\
\mathrm{nm}\end{array}$ \\
\hline \multicolumn{7}{|c|}{ BzFBz } \\
\hline S1 & - & 3.59 & - & 3.41 & 364 \\
\hline S4 & 324 & 4.28 & 437 & 4.27 & 290 \\
\hline \multicolumn{7}{|c|}{ BzFDp } \\
\hline S1 & 65 & 3.08 & 73 & 3.21 & 387 \\
\hline S3 & 151 & 4.10 & - & - & - \\
\hline S4 & 151 & 4.12 & - & - & - \\
\hline \multicolumn{7}{|c|}{ DpFDp } \\
\hline S1 & - & 3.38 & - & 3.10 & 400 \\
\hline S3 & 126 & 3.88 & 89 & 4.00 & 310 \\
\hline S5,6 & - & 4.01 & - & - & - \\
\hline S15 & 162 & 4.60 & - & - & - \\
\hline \multicolumn{7}{|c|}{ BzPFPBz } \\
\hline S1 & - & 3.06 & - & 3.08 & 403 \\
\hline S2 & 711 & 3.46 & - & - & - \\
\hline S4 & - & 3.99 & - & 4.00 & 310 \\
\hline S11 & 486 & 4.45 & - & - & - \\
\hline \multicolumn{7}{|c|}{ DpPFPBz } & 3.24 & 383 \\
\hline S1 & 154 & 2.83 & 162 & - \\
\hline S2 & 133 & 3.56 & - & 4.03 & 306 \\
\hline S6,7,8 & 445 & 4.25 & - & - & - \\
\hline
\end{tabular}


The SOS and CEO results (marked by solid lines on 2PA spectra in Fig.1) are in excellent quantitative agreement with each other, which provides a validation for the ATDA/SOS method. Predicted 2PA profiles also agree well with experimental ones. Experimental measurements of their spectral properties were reported in [14-16, 36]. While for most molecules agreement between resonant maxima is better than $0.1 \mathrm{eV}$, in the case of DpPFPBz theoretical bands are red-shifted relative to experimental

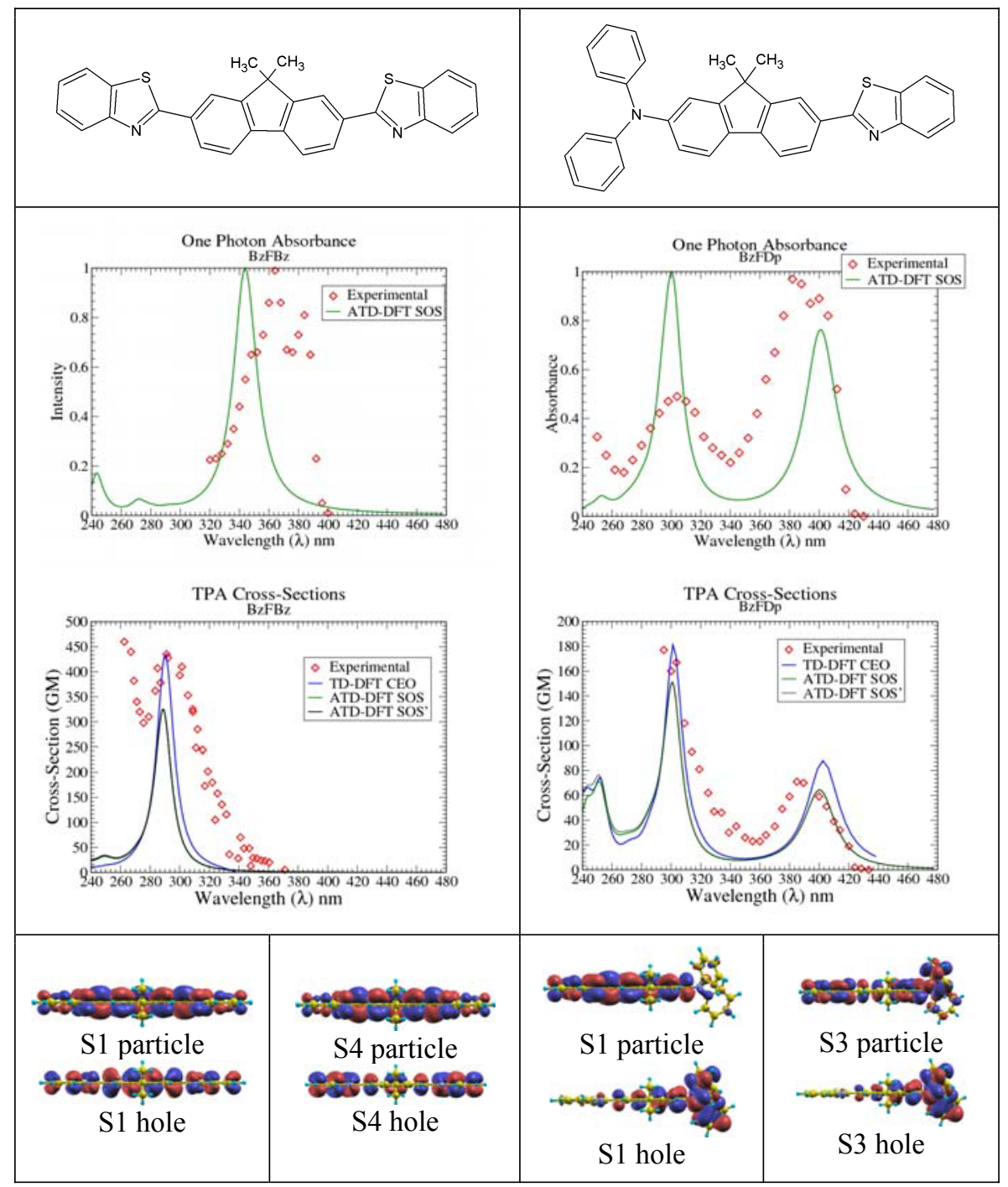

Fig. 1. Structural formulas (top row), 1PA profiles (row 2), 2PA profiles (row 3), and isosurfaces for natural transition orbitals (bottom row) for studied conjugated chromophores. Diamonds mark the experimental profiles; solid lines correspond to theoretical predictions with SOS and CEO formalisms. 
ones by approximately $0.44 \mathrm{eV}(55 \mathrm{~nm})$. This can be explained by greater conformational flexibility of the longer conjugated chain, and blue shift of the absorption spectra for non-planar conformations. Overall, agreement with experiment validates the use of TD-DFT as a part of rational design strategies directed toward new and improved two-photon absorbing materials.

\section{Conclusions}

We report theoretical study of five conjugated chromophores experimentally shown to have large two photon absorption cross-sections. We use the third order response formalism within Time Dependent Density Functional Theory to calculate frequencydependent second order hyperpolarizability in both the sum over states and coupled electronic oscillator formalisms to describe 2PA cross-sections. While CEO expressions do not lend themselves easily to a qualitative analysis, SOS ones can be simplified to essential state models and employed to identify $2 \mathrm{PA}$ resonant states and interpret the relationships between electronic structure and $2 \mathrm{PA}$ profiles.

We also use Natural Transition orbitals to compare the electronic structure of the linear and two-photon absorbing states. State to state transition dipole moments, necessary for SOS expressions are calculated with the a posteriori Tamm-Dancoff approximation and used to describe two-photon processes. Numerical values of the cross-sections obtained in SOS and CEO were found to be in good quantitative agreement with each other. This is the first time that TD-DFT/CEO and ATDA-DFT/ SOS methods have been compared for the calculation of 2PA spectra. Both CEO and SOS results are in good agreement with experiment. This validates the use of TD-DFT as a part of rational design strategies directed toward new and improved Two-Photon absorbing materials for bioimaging and optical data storage.

Acknowledgments. This work was supported in part by the National Science Foundation Grant No. CCF 0740344. The authors are thankful to DOE NERSC, UCF I2Lab, and UCF Institute for Simulations and Training (IST) HPC Stokes facility for the generous donation of the computer time. ST acknowledges support by the UCF NSTC and UCF Graduate Studies Office through the Summer Mentoring Research Fellowship. AEM acknowledges ACS COMP Hewlett-Packard Outstanding Junior Faculty award presented for this work at the Fall 2008 American Chemical Society Meeting.

\section{References}

1. Cumpston, B.H., et al.: Two-photon polymerization initiators for three-dimensional optical data storage and microfabrication. Nature 398, 51-54 (1999)

2. Kagotani, Y., et al.: Two-photon absorption and lasing due to biexciton in $\mathrm{CuCl}$ quantum dots. Journal of Luminescence 112, 113-116 (2005)

3. Zipfel, W.R., et al.: Live tissue intrinsic emission microscopy using multiphoton-excited native fluorescence and second harmonic generation. Proceedings of the National Academy of Sciences of the United States of America 100, 7075-7080 (2003) 
4. Rumi, M., et al.: Structure-property relationships for two-photon absorbing chromophores: Bis-donor diphenylpolyene and bis(styryl)benzene derivatives. Journal of the American Chemical Society 122, 9500-9510 (2000)

5. Wang, C.K., et al.: Effects of pi centers and symmetry on two-photon absorption cross sections of organic chromophores. Journal of Chemical Physics 114, 9813-9820 (2001)

6. Albota, M., et al.: Design of organic molecules with large two-photon absorption cross sections. Science 281, 1653-1656 (1998)

7. Belfield, K.D., et al.: Multiphoton-absorbing organic materials for microfabrication, emerging optical applications and non-destructive three-dimensional imaging. Journal of Physical Organic Chemistry 13, 837-849 (2000)

8. Belfield, K.D., et al.: Synthesis, characterization, and optical properties of new twophoton-absorbing fluorene derivatives. Chemistry of Materials 16, 4634-4641 (2004)

9. Ventelon, L., Moreaux, L., Mertz, J., Blanchard-Desce, M.: New quadrupolar fluorophores with high two-photon excited fluorescence. Chemical Communications, 2055-2056 (1999)

10. Kim, O.K., et al.: New class of two-photon-absorbing chromophores based on dithienothiophene. Chemistry of Materials 12, 284-286 (2000)

11. Fabian, J., et al.: Calculation of excitation energies of organic chromophores: a critical evaluation. Journal of Molecular Structure-Theochem 594, 41-53 (2002)

12. Hales, J.M., Hagan, D.J., Van Stryland, E.W., Schafer, K.J., Morales, A.R., Belfield, K.D., Pacher, P., Kwon, O., Zojer, E., Bredas, J.L.: Resonant enhancement of two-photon absorption in substituted fluorene molecules. Journal of Chemical Physics 121, 3152-3160 (2004)

13. Belfield, K.D., et al.: One- and two-photon fluorescence anisotropy of selected fluorene derivatives. Journal of Fluorescence 15, 3-11 (2005)

14. Belfield, K.D., Yao, S., Morales, A.R., Hales, J.M., Hagan, D.J., Van Stryland, E.W., Chapela, V.M., Percino, J.: Synthesis and characterization absorbing polymers of novel rigid two-photon. Polymers for Advanced Technologies 16, 150-155 (2005)

15. Belfield, K.D., et al.: Excited-state absorption and anisotropy properties of two-photon absorbing fluorene derivatives. Applied Optics 44, 7232-7238 (2005)

16. Schafer-Hales, K.J., Belfield, K.D., Yao, S., Frederiksen, P.K., Hales, J.M., Kolattukudy, P.E.: Fluorene-based fluorescent probes with high two-photon action cross-sections for biological multiphoton imaging applications. Journal of Biomedical Optics 10, 8 (2005)

17. Belfield, K.D., Schafer, K.J.: A new photosensitive polymeric material for WORM optical data storage using multichannel two-photon fluorescence readout. Chemistry of Materials 14, 3656-3662 (2002)

18. Kogej, T., Beljonne, D., Meyers, F., Perry, J.W., Marder, S.R., Bredas, J.L.: Mechanisms for enhancement of two-photon absorption in donor-acceptor conjugated chromophores. Chemical Physics Letters 298, 1-6 (1998)

19. Masunov, A.M., Tretiak, S.: Prediction of two-photon absorption properties for organic chromophores using time-dependent density-functional theory. Journal of Physical Chemistry B 108, 899-907 (2004)

20. Chen, G.H., Mukamel, S., Beljonne, D., Bredas, J.L.: The coupled electronic oscillators vs the sum-over-states pictures for the optical response of octatetraene. Journal of Chemical Physics 104, 5406-5414 (1996)

21. Cronstrand, P., Norman, P., Luo, Y., Agren, H.: Few-states models for three-photon absorption. Journal of Chemical Physics 121, 2020-2029 (2004)

22. Tretiak, S., Chemyak, V.: Resonant nonlinear polarizabilities in the time-dependent density functional theory. Journal of Chemical Physics 119, 8809-8823 (2003) 
23. Gel'mukhanov, F., Baev, A., Macak, P., Luo, Y., Agren, H.: Dynamics of two-photon absorption by molecules and solutions. Journal of the Optical Society of America B-Optical Physics 19, 937-945 (2002)

24. Baev, A., Prasad, P.N., Samoc, M.: Ab initio studies of two-photon absorption of some stilbenoid chromophores. The Journal of Chemical Physics 122, 224309-224306 (2005)

25. Ohta, K., Kamada, K.: Theoretical investigation of two-photon absorption allowed excited states in symmetrically substituted diacetylenes by ab initio molecular-orbital method. Journal of Chemical Physics 124, 124303-124311 (2006)

26. Cronstrand, P., Luo, Y., Agren, H.: Generalized few-state models for two-photon absorption of conjugated molecules. Chemical Physics Letters 363, 198 (2002)

27. Day, P.N., Nguyen, K.A., Pachter, R.: TDDFT study of one- and two-photon absorption properties: Donor-pi-acceptor chromophores. Journal of Physical Chemistry B 109, 1803 $1814(2005)$

28. Day, P.N., Nguyen, K.A., Pachter, R.: Calculation of two-photon absorption spectra of donor-pi-acceptor compounds in solution using quadratic response time-dependent density functional theory. Journal of Chemical Physics 125, 094103-094113 (2006)

29. Mikhailov, I.A., Tafur, S., Masunov, A.E.: Double excitations and state-to-state transition dipoles in pi-pi* excited singlet states of linear polyenes: Time-dependent densityfunctional theory versus multiconfigurational methods. Physical Review A 77, 012510 012511 (2008)

30. Badaeva, E.A., Timofeeva, T.V., Masunov, A.M., Tretiak, S.: Role of donor-acceptor strengths and separation on the two-photon absorption response of cytotoxic dyes: A TDDFT study. J. Phys. Chem. A 109, 7276-7284 (2005)

31. Cronstrand, P., et al.: Density functional response theory calculations of three-photon absorption. Journal of Chemical Physics 121, 9239-9246 (2004)

32. Frisch, M.J., et al.: Gaussian 98 Revision A.11 (1998)

33. Tretiak, S., Mukamel, S.: Density matrix analysis and simulation of electronic excitations in conjugated and aggregated molecules. Chemical Reviews 102, 3171-3212 (2002)

34. Martin, R.L.: Natural transition orbitals. Journal of Chemical Physics 118, 4775-4777 (2003)

35. Kokalj, A.: Computer graphics and graphical user interfaces as tools in simulations of matter at the atomic scale. Computational Materials Science 28, 155-168 (2003), http: // www. xcrysden.org/

36. Belfield, K.D., Bondar, M.V., Hernandezt, F.E., Przhonska, O.V., Yao, S.: Two-photon absorption cross section determination for fluorene derivatives: Analysis of the methodology and elucidation of the origin of the absorption processes. Journal of Physical Chemistry B 111, 12723-12729 (2007) 